\title{
Entrevista
}

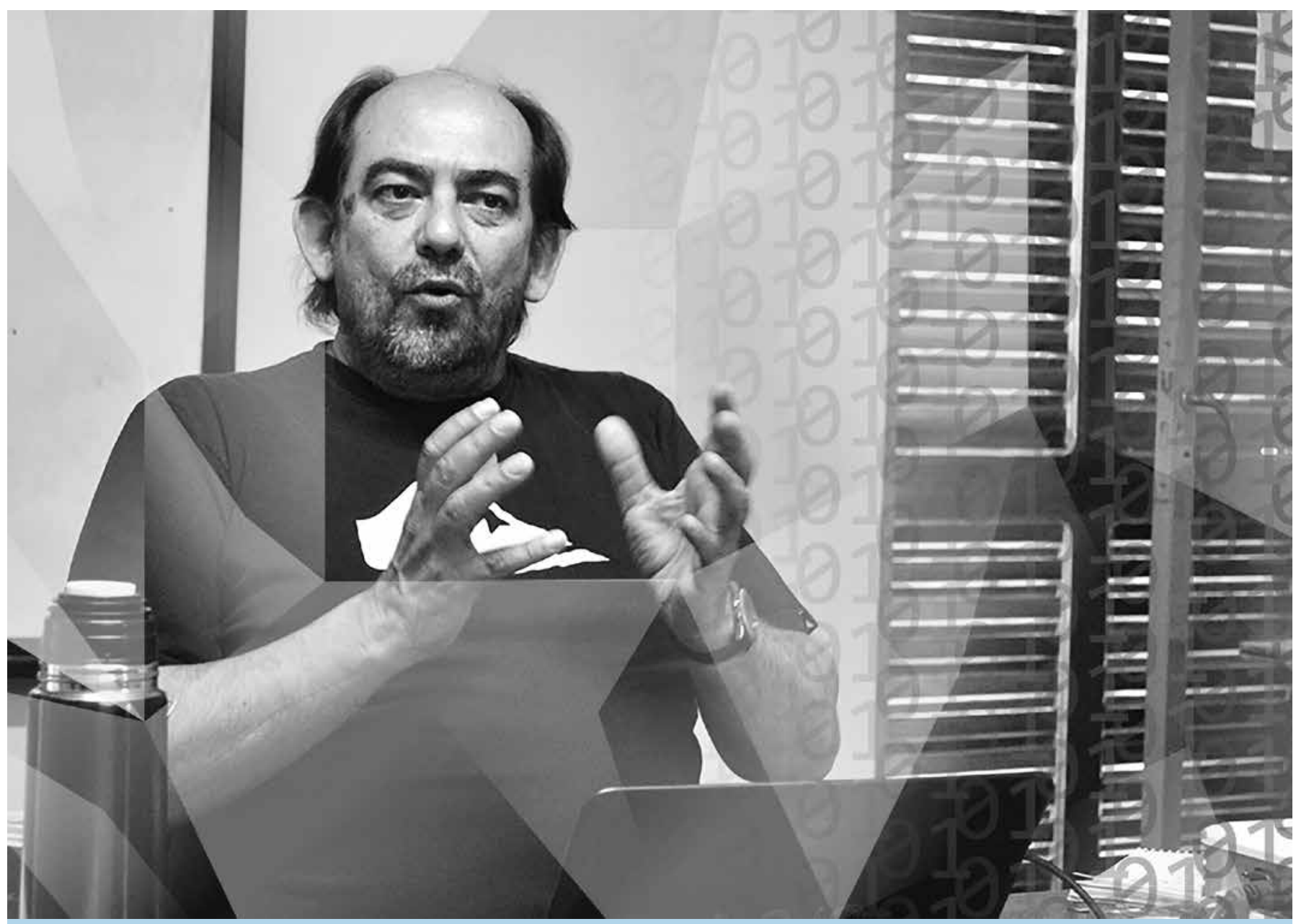

\section{Una conversación sobre los nuevos lenguajes desde la Comunicación Educación}

\section{Entrevista con Jorge Huergo}

Por: Sandra Patricia Ordóñez Castro

* (Nota de los editores:)"Esta entrevista al profesor Huergo es quizás una de las últimas que él concedió, por lo menos en el contexto colombiano. Lamentamos su reciente muerte y nos unimos a las voces de tristeza y reconocimiento de otros académicos y organizaciones de la comunicación-educación, la educación popular y comunitaria de América Latina, pues el aporte del profesor Huergo a estos campos, su compromiso político y su inmenso afecto y confianza en los jóvenes de los sectores populares nos dejó un legado de un campo de conocimiento entendido como praxis crítica y profundamente comprometida con la transformación social". 


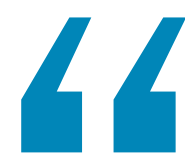

Con una vasta trayectoria coordinando proyectos comunicacionales y educativos junto a diferentes movimientos sociales y grupos comunitarios, el docente e investigador argentino Jorge Huergo, advierte sobre la importancia de hacer de los medios y las tecnologías en la escuela herramientas narrativas que favorezcan la comprensión de las dinámicas de la cultura, la multiplicación de las voces que la definen, y el protagonismo en su transformación. Superando el entusiasmo por la apropiación técnico-funcional de los nuevos lenguajes tecnológicos, la escuela debe apostar por una perspectiva comunicacional de su uso, potenciando el posicionamiento estratégico del sujeto como gestor de la cultura en la que vive.

¿Cuál sería desde una perspectiva educativa el papel de los nuevos lenguajes tecno-digitales en la configuración contemporánea de la cultura y del conocimiento?

Creo, en primer lugar, que hay algunas cuestiones que no están suficientemente estudiadas. Pienso, por ejemplo, en el caso de Argentina, en Conectar igualdad, que es un programa de educación uno a uno desde el concepto del netbook. Me parece que todavía no se han estudiado suficientemente sus impactos, ni las transformaciones que genera a nivel cultural, en relación con la apropiación de conocimientos, etc. Me parece que allí hay un pro y un contra: es decir, hay toda una fascinación en torno a la multiplicación de formas de acceso al conocimiento, de posibilidades que tienen que ver con una mayor acumulación y distribución de la información. Pero como contracara, me parece que hay algo que es clave, y es hasta qué punto la información se transforma en conocimiento.

Desde las estrategias educativas nosotros admitimos esas nuevas dinámicas, las reconcentramos o las retraemos a aquello que ha sido la obsesión de la escolarización por muchos siglos, y que tiene que ver con lo que Paulo Freire refería como la búsqueda de "las maneras de disciplinar la entrada del mundo en la consciencia”. Entonces, yo creo que antes de obsesionarnos por disciplinar la entrada del mundo en la consciencia, sería muy bueno pensar, desde la educación, de qué modo, potenciando las tecnologías, y el sentirse sujeto protagonista de una cultura profundamente modificada por los medios tecnológicos, podemos desarrollar comprensiones críticas acerca del mundo en que vivimos y de las formas de actuar en él. Esa es una forma de ciudadanía que es muy concreta en términos educativos en el día de hoy. ¿Existe algún límite entre el uso/divertimento tecno-digital, y la dinamización de la cultura?, ¿cuál es el lugar de la escuela en este acoplamiento?

Yo creo que el límite básico está interpuesto por lo que son las referencias de la dignidad y de la diversidad en la vida huma- na. Me parece que hay algo que es clave: admitir las diferencias, las diferentes voces, y también poder recorrer las historias por las cuales se han venido fraguando esas diferencias, que muchas veces es una historia de desigualdad. Esto tiene que mediar de alguna manera el acceso a las industrias culturales y su uso. Lo que la escuela tiene que aportar, fundamentalmente, es todo aquello que nos ayude a comprender y actuar en la cultura en la cual vivimos: antes que rechazarla. Antes que ponerla en tela de juicio en función de parámetros de otros momentos de la historia, creo que la escuela tiene que provocar espacios en los cuales pueda ser expresada y reconfigurada la comprensión del tipo de experiencia que realizamos, del tipo de modernidad que adquiere lo social, la creatividad, la estética, etc., en el mundo que vivimos. Usted lleva más de 15 años liderando debates en el campo de la Comunicación/Educación y trabajando con diferentes organizaciones sociales: ¿qué es lo que se ha venido gestando en este campo en Latinoamérica y cuál es el lugar de los nuevos lenguajes tecnológicos en el proceso?

Bueno, efectivamente, hace ya 20 años que vengo trabajando en esto y puedo decir que la relación entre Comunicación y Educación ha ganado un espacio académico y se han generado procesos de identificación con determinadas corrientes. Eso ha sido muy importante. Pero también, como contracara, ha habido la tendencia (fundamentalmente por la marca del neoliberalismo), a reducir el campo a la relación entre las tecnologías y la educación: una especie de entusiasmo por tecnificar la escuela y por trabajar pedagógicamente mediante el uso de las tecnologías. Yo creo que allí ha habido una mala comprensión de lo que significa la mediatización de la cultura. Desde mi experiencia con los maestros, me parece que ellos no acaban de comprender e incluso rechazan la mediatización de la cultura, pero como el uso de las tecnologías se puede reducir al campo de la didáctica, entonces eso les va más o menos bien. Creo que ese es uno de los desafíos que nos quedan por recorrer. 
En estos 20 años también he visto que, en ese esfuerzo por comprender y realizar prácticas relacionadas con la vinculación entre tecnologías y educación, se llega a perder de vista el carácter estratégico político de nuestro campo. Es decir, yo creo que el campo de Comunicación/Educación no sólo nació como espacio estratégico político, sino que se fue desarrollando y adquiriendo sus propias formas, sobre todo en lo que uno podría llamar la Comunicación/Educación popular.

En este sentido, lo que yo veo como peligro es que, en los tiempos que uno podría considerar de restitución del Estado, de restitución de lo público, luego de las dictaduras y del neoliberalismo en muchos países de América Latina, en la academia nos estemos quedando al margen de toda esa riqueza de la Comunicación/Educación popular. Por eso creo que hay dos ejes para trabajar: por una parte, la distribución y apropiación de tecnologías y medios en la educación y, por otra, la vinculación de la Comunicación/ Educación en los sectores populares, en los movimientos sociales, en las formas de lucha por el reconocimiento en la sociedad. ¿Cómo cree usted que los lenguajes de los medios y tecnologías pueden potenciar la emancipación como objetivo político de la práctica educativa?

Yo creo que, fundamentalmente, al posibilitar la expresión de diferentes voces (esto tiene que ver con la tradición de la Pedagogía Crítica o de la Liberación, que está muy presente en el pensamiento de Freire, de Peter McLaren, etc.): la posibilidad de expresión de las diferentes voces, las diferentes visiones del mundo, los diferentes quehaceres, las diferentes escenas de lucha por el reconocimiento, las diferentes identidades, etc. Una vez instalada esta posibilidad, la educación tiene mucho por hacer en términos de poder asumir, a veces incluso, las características conflictivas que adquiere el pronunciamiento de diferentes voces, en un recorrido que sea no violento. En una sociedad "mundializada" es muy importante que el ciudadano sea capaz de abordar los conflictos que se producen en la vida social, sin querer solucionarlos prematuramente, ni pretender destruir al otro.

Desde su experiencia de trabajo en comunicación popular comunitaria, ¿cómo pueden los medios y tecnologías, potenciar en la escuela formas de comunicación no hegemónicas?

Yo creo que lo no hegemónico pasa necesariamente por la creación de espacios de reflexión y de creatividad de los mismos sujetos que actúan cotidianamente en la educación. No hay recetas para eso, no hay cabezas iluminadas, pero me parece que el Estado lo que debe hacer es garantizar espacios donde los estudiantes y los docentes puedan de algún modo construir nuevas narrativas. Es en este sentido que hay que repensar la vinculación entre tecnologías y educación.

Hay mucho que se puede recuperar a ese nivel en las tradiciones no hegemónicas latinoamericanas y hay también algunas tradiciones europeas que me parece que tienen perfecta vigencia todavía, como la tradición de Célestin Freinet, el pedagogo francés que utilizó por primera vez el periódico en la escuela. Eso sirvió para que los niños investigaran sobre su realidad, más allá de las informaciones que se producían al respecto (que en el caso de la sociedad actual muchas veces están mediadas por intereses políticos, económicos, etc.). Es el asunto de salir a terreno, de encontrarse con la experiencia cotidiana. Eso es lo que hay que potenciar: la presencia de la tecnología y de los medios en el ámbito educativo tiene que servir para que los niños vayan formándose en una especie de "protagonismo popular", podríamos decir, en una especie de protagonismo en su formación ciudadana.

¿Hablaríamos entonces de un desplazamiento en lo educativo en donde la materia de enseñanza es una continuación de la experiencia?, ¿quién es entonces el maestro, y qué vendría siendo la escuela?

Efectivamente. Y por eso debemos reforzar la idea de un docente que se transforme en pedagogo: un docente que pueda reflexionar y narrar desde un punto de vista conceptual e incluso teórico, su propia práctica; pero además, y fundamentalmente, un docente que se asuma como trabajador de la cultura. Es decir, el que al potenciar la experiencia generadora, puede reconocerse como hijo de una cultura, pero también como gestor de la misma, como su transformador y protagonista. Ese es un trabajo en el cual necesariamente tiene que acompañar a los niños. Los padres generalmente no tienen esa visión que va más allá de lo que forma parte de su propia experiencia, y me parece que uno de los objetivos fundamentales de la pedagogía es precisamente la incorporación crítica en el mundo de la cultura.

Por lo mismo pienso que la escuela hay que repensarla. Nosotros todavía tenemos experiencias escolares muy ligadas a lo que fue el disciplinamiento de la época de la organización nacional. Tenemos todavía mucho de expectativas de normatización de saberes a través de las disciplinas. El papel de la escuela no es solamente la transmisión de cultura y de saberes, sino de una dimensión crítica que dé la posibilidad de desnaturalizar esa cultura y esos saberes (fundamente buscando aquellas cuestiones que son negaciones para la vida humana) y problematizar, es decir, reencontrarnos con nuevas condiciones de posibilidad para la producción y la creación cultural. 


\section{No podemos circunscribir todo el espectro de las nuevas situaciones que se producen en la cultura a la formación docente, no podemos hacerlo solamente a través de la creación de espacios en los que los docentes aprendan a utilizar los medios desde el punto de vista didáctico. Hay mucho más que eso: hay que apuntarle al posicionamiento protagónico de los docentes en estas nuevas coordenadas culturales.}

Allí hay algo del orden de la formación de los maestros que es imprescindible trabajar: no podemos circunscribir todo el espectro de las nuevas situaciones que se producen en la cultura a la formación docente, no podemos hacerlo solamente a través de la creación de espacios en los que los docentes aprendan a utilizar los medios desde el punto de vista didáctico. Hay mucho más que eso: hay que apuntarle al posicionamiento protagónico de los docentes en estas nuevas coordenadas culturales.

¿Cómo solventar el riesgo de circunscribir el uso de los nuevos lenguajes y tecnologías al terreno de la didáctica, y lograr posicionar al docente en este nuevo nivel de ejercicio cultural haciendo uso de las mismas?

Entendiendo que el maestro no es solamente alguien que sabe enseñar, sino que es alguien que tiene que poder trabajar con la cultura en la que vive y que tiene que producir impulso pedagógico. No pensar tanto en la dotación de nuevos equipamientos o dispositivos, y más bien hacerlo a favor de aportar a la creación y a la inscripción en cuestiones que están presentes en la sociedad de múltiples maneras, ahora con la mediación de las tecnologías.

La idea sería entonces lograr que los actores del escenario educativo pudieran valerse de estos nuevos lenguajes en el propósito de conocer, relatar y transformar su entorno y asumir su ciudadanía de manera activa...

Sí, lo cual implica empezar a entender la educación en otros ámbitos. Hay una cuestión mucho más fuerte que la transmisión de conocimientos, que tiene que ver con cómo formamos la subjetividad. Y la subjetividad se forma también con nuevos repertorios lingüísticos, es decir, potenciando nuevas formas de comprender y de hacer posible la experiencia.

La escuela ha perdido en gran medida su carácter interpelador, y lo ha hecho por desconocer otras formas de educación que están presentes en la sociedad. La subjetividad tiene mucho que ver con las posibilidades de contar y de contarse, es decir, con lo que Paul Ricoeur llamaba la identidad narrativa. Nuestras escuelas deben ser capaces de promover y de articularse con las formas de narración de la identidad que se están produciendo en la sociedad, para poder cumplir con su tarea fundamental, que es inscribir a los niños en la forma de sociedad en la que viven. ¿Qué hacer entonces, en términos de la formación docente, para posibilitar este tipo de impulso pedagógico más allá de la tradición contenidista, en un sentido social e histórico?

Bueno, yo tuve que coordinar la formación docente en la provincia de Buenos Aires, que es una de las jurisdicciones más grandes de América Latina: tenía a mi cargo 211 instituciones de formación docente y desde allí produje una transformación con la que la gente está muy comprometida. Se trata de la inscripción de la escuela en un campo educativo. Es decir, pensar la escuela en contexto.

Para esto lo que propusimos y fuimos logrando fue la formación de los maestros, no sólo en la tarea escolar, sino también en organizaciones sociales. Implementamos como mínimo un año en formación en organización de la comunidad. Este es un componente que tiene que ver con las condiciones conflictivas y complejas de nuestras sociedades, para las cuales el maestro muchas veces no está preparado. La idea es, en primer lugar, debilitar las fronteras entre la escuela y su contexto de influencia, y, por otro lado, lograr que el maestro comprenda y asuma un posicionamiento estratégico frente la complejidad y las conflictivas que son propias de la sociedad toda, y que se instalan en la escuela. ¿Cuáles son las rutas concretas de implementación de este modelo?

Lo primero es crear las condiciones para una experiencia social del maestro en formación. Eso es lo central. La segunda cuestión es construir espacios que permitan integrar la práctica con la teoría y el conocimiento con el mundo. Nosotros creamos talleres integradores interdisciplinarios enfocados en problemas concretos que convocan, desde su centro, la posibilidad de intervención conjunta de las disciplinas. Yo creo que esa es la estrategia en formación docente que ofrece una alternativa frente a la centralidad en la didáctica. 
Desde esta perspectiva, ¿podríamos pensar que el objeto de la educación sería la subjetivación política del estudiante (ese asumir su lugar en el mundo y agenciar su proyecto de vida)?

Sí. En gran medida es eso. Pero no podemos tampoco vaciar a la educación de contenido. Los conocimientos relacionados con la cultura establecida y los saberes en su versión educativa (que no son tampoco los saberes de la ciencia), son muy importantes. Pero hay que atender a un propósito y saber cuál es la incertidumbre en el logro de ese propósito: búsquedas, dudas y angustias, muchas veces. Entender esto implica en ocasiones recurrir a formas de lo educativo que no están diseñadas de antemano. ¿Y en ese proceso la comunicación podría entenderse como epistemología?

Sí. La comunicación, más que como una ciencia, como una práctica y como una experiencia. A mí, por lo menos, lo que me interesa de lo comunicacional es eso. Sí es importante tener algunas claves para comprender desde el punto de vista conceptual los procesos de la comunicación, pero me parece que lo clave es adentrarse en la experiencia. Cuando uno trabaja con el eje puesto en la cultura mediático-tecnológica, y no en las tecnologías como didáctica de la educación, de lo que se trata es de comprender las transformaciones que se han producido en nuestra cultura a partir de los medios y las tecnologías, y no simplemente pensar en cómo usarlos.

Por supuesto, también importante poder, digamos "gobernarlos" con una finalidad educativa. Pero lo fundamental es captar desde un punto de vista flexible cuáles son las transformaciones en procesos que se están produciendo. Es aquello que decía Jesús Martín Barbero: a costa de perder los objetos, tenemos que ganar los procesos. Nuestro entorno se transforma hoy en día de manera incesante, y eso produce inestabilidad, transitoriedad, multiplicación, diseminación...

Una transformación de la cultura, que es al mismo tiempo una mutación de las formas de pensar/construir/comunicar sentido. Esta transformación configura "una lógica" (una "poética" si se quiere, en el sentido aristotélico, vinculada a esa inestabilidad, transitoriedad, multiplicación, etc.), que si bien surge en el uso tecnológico, se puede entender más allá del medio-de-uso, y traducir en función de cualquier experiencia. ¿No comprender esto podría suponer un riesgo en el propósito educativo?

En este mundo que ha sido transformado por tecnologías y medios en el uso cotidiano, hay nuevas condiciones del saber, nuevas condiciones del conocimiento, una fuerte confusión entre información y conocimiento, pero bueno... los maestros tienen sentido común... Después lo que viene es considerar las nuevas formas de socialización, de la sexualidad, de la lectura cotidiana del mundo, sin dejar de lado los vínculos entre pares en el barrio, o con una determinada organización social o un movimiento ideológico. Lo que creo es que el desplazamiento de esta "lógica" no se ha estudiado lo suficiente y que es necesaria una investigación general e integrada en Comunicación/Educación que no desconozca ninguno de los dos aspectos de constitución de la realidad, y que comience a evidenciar sus vínculos.

¿El camino de esa investigación podría ser el de una hermenéutica interpretativa transversal, enfocada fundamentalmente en los relatos?

Yo creo que sí, como parte de las nuevas condiciones de reconocimiento de lo cultural. Y esa hermenéutica o esa comprensión de lo que está sucediendo, tiene que ser integral. Ahí hay algo de lo que decía Martin Heidegger: para poder actuar con libertad (resaltando esta palabra "poder", no me gusta la palabra, pero para "empoderarnos"), necesitamos comprender. Esto, claro, es algo que se va dando de manera articulada. No hay que alcanzar toda la comprensión para luego desarrollar un poder actuar, sino que en el poder actuar también se va articulando la comprensión. La educación debe potenciar esta dinámica para ajustar su ejercicio a las nuevas realidades y para favorecer el surgimiento de nuevas narrativas de identidad, así como su reconocimiento.

\footnotetext{
La subjetividad tiene mucho que ver con las posibilidades de contar y de contarse, es decir, con lo que Paul Ricoeur llamaba la identidad narrativa. Nuestras escuelas deben ser capaces de promover y articularse con las formas de narración de la identidad que se están produciendo en la sociedad, para poder cumplir con su tarea fundamental, que es inscribir a los niños en la forma de sociedad en la que viven.
} 
Tema: Nuevos Lenguajes y Educación

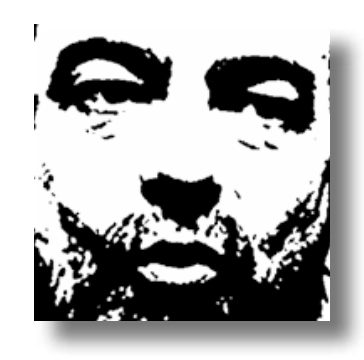

